\title{
Human Papillomavirus Vaccination and Physical and Mental Health Complaints Among Female Students in Secondary Education Institutions in Denmark
}

\author{
Tatjana Gazibara, MD, PhD ${ }^{1,2}$, Lau Caspar Thygesen, PhD ${ }^{7}$, Maria Holst Algren, PhD ${ }^{7}$, \\ and Janne Schurmann Tolstrup, $P h D^{7}$
}

${ }^{1}$ National Institute of Public Health, University of Southern Denmark, Copenhagen K, Denmark; ${ }^{2}$ Institute of Epidemiology, Faculty of Medicine, University of Belgrade, Visegradska 26A, Belgrade, Serbia.

\begin{abstract}
BACKGROUND: Previous studies have not explored the effect of HPV vaccination on health status at a longer time interval. Similarly, self-reported physical and mental health in recipients of the HPV vaccine has not been studied.
\end{abstract}

OBJECTIVE: To evaluate whether HPV vaccination was associated with physical and mental health complaints among girls in secondary education institutions.

DESIGN: Prospective cohort study.

PARTICIPANTS: This study used data from girls aged 1520 years who participated in the Danish National Youth Study (DNYS) 2014. Data on HPV vaccination was retrieved from the Danish Vaccination Register.

MAIN MEASURES: Participants were asked whether they had experienced headaches, stomachache, neck pain, menstrual cramps, sore throat, sadness, irritation, nervousness and sleep problems in the past 6 months.

KEY RESULTS: Of 41,333 girls, 39, 145 (94.7\%) received at least one dose of HPV vaccine. The most prevalent health complaint among the vaccinated and not vaccinated females was "been irritable" (88.2\% and 88.4\%, respectively). The lowest proportion of health complaints was stomachache $(49.6 \%$ in vaccinated and in 50.4\% in unvaccinated girls). Regression model, adjusted for sociodemographic characteristics and health behavior, showed that HPV vaccination was associated with a lower likelihood of reporting sore throat (odd ratio [OR] 0.86, 95\% confidence interval [CI] 0.78-0.95) and being sad (OR 0.86, 95\%CI 0.76-0.97). Similar results were observed when HPV vaccination status was analyzed according to the number of doses received.

CONCLUSION: We conclude that HPV vaccination was not associated with physical and mental health complaints among girls in secondary education institutions in Denmark after a median of 5.3 years since HPV vaccination.

KEY WORDS: HPV vaccination; girls; secondary education institutions; health status.

\begin{tabular}{l}
\hline Prior Presentations The results of this study are presented in a poster \\
session of the 9th Nordic Conference of Epidemiology and Register-Based \\
Health Research, 18-20 September 2019, Tampere, Finland.
\end{tabular}

Electronic supplementary material The online version of this article (https://doi.org/10.1007/s11606-020-05845-8) contains supplementary material. which is available to authorized users.

Received November 29, 2019

Accepted April 7, 2020

Published online April 27, 2020
J Gen Intern Med 35(9):2647-54

DOI: $10.1007 / \mathrm{s} 11606-020-05845-8$

(c) Society of General Internal Medicine 2020

\section{INTRODUCTION}

Bivalent and quadrivalent human papillomavirus (HPV) vaccines were approved for use in Denmark in October 2006 and since 2009 HPV immunization has been a part of the national immunization program. ${ }^{1}$ Girls aged 12 years were targeted for HPV vaccination, while a catch-up program was also established for girls and women until the age of $26 .{ }^{1}$ The HPV vaccine initiation coverage rates were initially above $80 \%$ following the introduction of the program; however, the coverage dropped in the period 2014-2016 due to case reporting of adverse effects after HPV vaccination by the popular Danish media. ${ }^{2}$ Most adverse effects accounted for postural orthostatic tachycardia syndrome (POTS), headache, fatigue, nausea, segmental dystonia, cognitive impairment, and neuropathic pain. ${ }^{3}$ After this period, following a national campaign aiming at informing the public about HPV vaccination in 2017-2019, the vaccine initiation rates have increased and are now comparable with those before the reports of adverse vaccination effects. $^{4}$

Register-based studies in large Nordic population samples reported that HPV vaccination was not associated with a higher risk of developing neurological and autoimmune diseases, ${ }^{5}$ chronic fatigue syndrome, ${ }^{6,7}$ complex regional pain syndrome, POTS, ${ }^{7}$ or adverse pregnancy outcomes. ${ }^{8}$ A recent report from the World Health Organization highlighted that the HPV vaccine is safe and effective in spite of low worldwide uptake. ${ }^{9}$ Furthermore, register-based analyses in Denmark suggested that girls and women who reported adverse effects following vaccination had used health-care services more frequently before vaccination compared with girls and women who did not report post-vaccination adverse effects indicating that for some of the women, reported adverse effects were due to pre-existing health conditions. ${ }^{10,11}$

Adolescence is characterized by intense physical and psychological changes. While most adolescents are in good health, somatic and psychological symptoms do occur. 
Although these symptoms can be suggestive of specific chronic illnesses, they may also present independently "as imbalance between the increasing educational, social, and sports demands on young people and physiological 'debts' owed to rapid growth and sexual development". ${ }^{12}$ Somatic symptoms frequently experienced by adolescents account for headache, stomachache, and backache. Chronic fatigue syndrome in adolescence includes among others sore throat, muscle discomfort or pain, headaches and sleep problems. ${ }^{12}$ Of mental health problems, mood swings, irritability, anxiety, and depression have commonly been addressed. ${ }^{13}$ Because most adolescents do not report to their physician for physical and mental health complaints, ${ }^{12}$ it is difficult to analyze potential factors that contribute to their onset.

Previous studies using nationwide administrative systems for reporting of adverse effects suggested that there has been no evidence of the effect of HPV vaccination on health status at a longer time interval. Similarly, self-reported physical and mental health in recipients of the HPV vaccine has not been studied. Registers contain information on events that require hospital discharge or prescription medication. Although register-based studies offer complete data on a whole population, it is often difficult to adjust predefined exposures (such as vaccination status) for important characteristics associated with vaccination compliance. Due to the subjective nature of physical and mental health complaints, their recognition requires specific data collection. As a result, assessment of health complaints using only register-based data can be insufficient. Therefore, using self-reported outcomes approach in the target population could offer an opportunity for a more detailed insight into the issue. The purpose of this study was to evaluate whether HPV vaccination was prospectively associated with physical and mental health complaints in a large prospective cohort study among girls in secondary education institutions in Denmark.

\section{METHODS}

\section{Participants}

Data for this study were extracted from the Danish National Youth Study 2014 (DNYS). ${ }^{14}$ The purpose of DNYS was to study physical and mental health and health-related behavior among students at the secondary education level in Denmark. Invitation for participation was sent out to 137 general high schools and 12 largest vocational schools, of which 119 general high schools and 10 vocational schools provided consent for participation. Students from all grades and classes within high schools were invited to participate $(N=83,751)$, whereas in vocational schools students in basic courses were invited $(N=7527)$. Student response rate among the participating general high schools was $87 \%(70,674)$, and among vocational schools, it was $69 \%$ (5179), yielding a total of 75,853 students in the sample. Of those, 71,835 students (94.7\%) were linked to their unique personal identification number (CPR) allowing linkage of DNYS data with the Danish Civil Registration System as well as with the Danish Vaccination Register (DVR) ${ }^{15}$ Of 71,835 students, only girls aged $15-20$ years $(41,333)$ were included in this analysis. Selection of girls only was based on the circumstance that at the time of survey, HPV vaccination was recommended only to girls. Age range 1520 years was selected because the DNYS covered a small percentage $(2.72 \%)$ of women above the age of 20 who were in secondary education institutions, and were likely not regular students, but rather needed to catch up on the period when they were away from school.

\section{Data Collection}

Data on HPV vaccination was retrieved from the DVR retrieving Anatomical Therapeutic Chemical [ATC] code J07BM01, because the four-valent HPV vaccine was used from October 2006 to 2014 in Denmark. The number of doses and the dates of vaccination were collected for all vaccinated students from October 2006 to the date of the survey in 2014. The majority of HPV vaccination records (>90\%) showed at a minimum 4week period between the first and the second dose and at least an 8 -week period between the second and the third dose. The variable HPV vaccination was classified as "unvaccinated" if a person did not have any record of HPV vaccination (0 doses) and "vaccinated" with 1, 2 or 3 doses. If records showed more than three doses, these individuals were categorized as having received three doses of the HPV vaccine.

Participants filled in an electronic questionnaire. Participants were asked whether they had experienced the following in the past 6 months: headaches, stomachache, neck pain, menstrual cramps, sore throat, sadness, irritation, nervousness, and sleep problems. Answers were graded on a 5-point scale: 1 (almost every day); 2 (more than one time per week); 3 (every week); 4 (almost every month); 5 (rarely or never). Answers were classified as a binary outcome variable: value 0 was assigned to answer "rarely or never" and value 1 was assigned to all other answers. To check physical and mental health complaints at a higher frequency, such as at least 2 times per week each week in the past 6 months, answers were also classified as a binary outcome variable based on these cutoffs: value 0 was assigned to answers "rarely or never," "almost every month," and "every week" and value 1 was assigned to answers "more than one time per week" and "almost every day." This outcome was labeled as "weekly physical and mental health complaints." These somatic and mental health symptoms were selected because they were previously listed as important symptoms in adolescent medicine. ${ }^{12,13}$ Menstrual cramps were added to account for complaints related to menstruation.

Information on socio-demographic characteristics (age, gender, ethnicity, living arrangements) and health behavior (smoking, alcohol, drug use, and physical activity were included in this study) were collected using a questionnaire. A detailed description of the variables is provided in 
the Supplemental Methods in the ESM. Ethnicity was classified as Danish (if both parents were Danish); Danish and other (one parent was Danish and the other parent was of another ethnicity), and other than Danish (if both parents were of another ethnicity than Danish). Living arrangements were classified based on whether the student lived with both or one parent or had other living arrangements. Daily smoking was categorized as "yes vs. no." Alcohol intake was classified based on students' estimated intake of alcoholic drinks per week (according to a chart that explained how alcohol units were calculated). Drug use was related to ever vs. never use of hash/cannabis. Physical activity was related to the practice of sports or other physical activity that induces sweating, excluding low-speed cycling. This variable was categorized as yes vs. no.

After linkage of DNYS 2014 data of the participants with their respective CPRs, every student was assigned a specific 12-figure code. This code differed from their CPR and allowed for anonymization of the database. Because of this, it was impossible to de-identify participants and track their data back to their corresponding CPRs.

Prior to filling in the questionnaire, participants were asked to read and accept an informed consent form that clearly expressed that participation was voluntary and that individual data would be kept confidential. In Denmark, the age of 13 years is the minimum age at which an individual can consent to processing of personal information. For this reason, the consent from the parents was not required. ${ }^{16}$ Data collection was approved by the Danish Data Protection Agency J. No. 2013-54-0526. Participants signed informed consent for participation.

\section{Data Analysis}

Descriptive measures (count and percentages) were calculated. Logistic regression models were used to evaluate the associations between ever HPV vaccination and each of 10 selfreported physical and mental health complaints. In all models, the dependent variable was the self-reported physical or mental health complaints and the independent variable was HPV vaccination status. The series of logistic models included the following: (1) Crude model only including HPV vaccination status; (2) Partially adjusted model included age, ethnicity, type of schoo, and living arrangements as covariates; and (3) Fully adjusted model further included smoking status, alcohol use, drug use and physical activity as additional covariates.

In sensitivity analysis, we stratified the sample according to the median length of time since vaccination. Because adverse effects of vaccination are expected after shorter periods since vaccination, the purpose of this analysis was to examine the association between receiving at least one dose of the HPV vaccine and physical and mental complaints among girls who reported complaints after a shorter and longer time since vaccination.
Data analysis was performed in SAS, version 9.4. Graphic display was performed in $\mathrm{R} .{ }^{17}$ Effect estimates were presented as odds ratios (OR) with corresponding $95 \%$ confidence intervals $(95 \% \mathrm{CI}) . P$ values below 0.05 were considered statistically significant.

\section{RESULTS}

Out of 41,333 female students, 39,145 (94.7\%) received at least one dose of the HPV vaccine. Socio-demographic characteristics of girls according to vaccination status are shown in Table 1. Most girls in our sample were of Danish ethnicity and living with both parents (Table 1). The majority of girls were non-smokers, drank 1-6 or 7-13 alcohol units per week, did not report illicit drug use, and were engaged in weekly physical activity (Table 1). Mean and median length of time between HPV vaccine initiation and taking the survey were 5.1 and 5.3 years respectively (time since vaccination range 0.9 8.0 years).

The most prevalent health complaint among the vaccinated and not vaccinated girls was "been irritable" (Table 2). The lowest proportion of health complaints was stomachache (Table 2). In the crude logistic regression model, girls who were vaccinated were less likely to report sore throat, but more likely to be irritable (Table 2). In the partially adjusted model, the girls who were vaccinated were less likely to report sore throat and (OR 0.86, 95\% CI 0.78-0.95) and being sad (OR $0.86,95 \%$ CI 0.76-0.97) (Table 2).

A similar pattern was observed when weekly physical and mental health complaints were analyzed. The crude model showed that girls who received HPV vaccination were less likely to experience weekly sadness, nervousness and sleep problems (data not shown).

When HPV vaccination status was analyzed according to doses $(1,2$, and 3 doses vs. 0 ), fully adjusted model showed null association with all self-reported physical and mental health complaints, except for sore throat and being sad. Specifically, receiving three HPV vaccine doses was associated with a lower likelihood of reporting sore throat and (OR 0.86, 95\% CI 0.77-0.95) and being sad (OR 0.85, 95\%CI 0.76 0.97) (Table 3).

The results of sensitivity analysis are shown in Fig. 1. In girls who received at least one dose of HPV vaccine $\leq 5.3$ years ago, HPV vaccination was associated with a lower likelihood of reporting sore throat in the past 6 months (OR $0.80,95 \%$ CI 0.71-0.91). In girls who received at least one dose of HPV vaccine more than 5.3 years ago no association between HPV vaccination and physical or mental health complaints was observed (Fig. 1).

\section{DISCUSSION}

The findings of this study suggest that having received at least one dose of HPV vaccine was not associated with physical and 
Table 1 Socio-demographic and Health Behavior Characteristics Regarding the HPV Vaccination Status Among Girls in Secondary Education Institutions in Denmark $(N=41,333)$

\begin{tabular}{|c|c|c|}
\hline \multirow[t]{2}{*}{ Characteristics } & \multicolumn{2}{|l|}{ HPV vaccination status } \\
\hline & Vaccinated $N=39,145$ n $(\%)$ & Unvaccinated $N=2188$ n $(\%)$ \\
\hline \multicolumn{3}{|l|}{ Age (years) } \\
\hline $15-16$ & $8386(21.4)$ & $460(21.0)$ \\
\hline 17 & $13,370(34.1)$ & $712(32.5)$ \\
\hline 18 & $11,689(29.8)$ & $678(31.0)$ \\
\hline $19-20$ & $5700(14.6)$ & $338(15.5)$ \\
\hline \multicolumn{3}{|l|}{ Ethnicity } \\
\hline Danish & $35,637(92.0)$ & $1643(78.0)$ \\
\hline Danish and other & $2367(6.1)$ & $287(13.6)$ \\
\hline Other than Danish & 749 (1.9) & $178(8.4)$ \\
\hline Missing & $356(0.9)$ & $80(3.6)$ \\
\hline \multicolumn{3}{|l|}{ Type of school } \\
\hline High school & $38,527(98.4)$ & $2125(97.1)$ \\
\hline Vocational school & $622(1.6)$ & $63(2.9)$ \\
\hline \multicolumn{3}{|l|}{ Living arrangement } \\
\hline With both parents & $25,102(64.6)$ & $1220(56.8)$ \\
\hline With one parent & $11,366(29.3)$ & $667(31.0)$ \\
\hline Other & $2381(6.1)$ & $261(12.2)$ \\
\hline Missing & $296(0.8)$ & $40(1.8)$ \\
\hline \multicolumn{3}{|l|}{ Smoking } \\
\hline Yes & $4198(10.7)$ & $275(12.7)$ \\
\hline No & $34,846(89.2)$ & $1894(87.3)$ \\
\hline Missing & $101(0.3)$ & $19(0.9)$ \\
\hline \multicolumn{3}{|c|}{ Alcohol use in units per week } \\
\hline 0 & $2442(7.0)$ & $169(9.8)$ \\
\hline $1-6$ & $11,200(32.0)$ & $611(35.4)$ \\
\hline $7-13$ & $12,064(34.5)$ & $504(29.2)$ \\
\hline$\geq 14$ & $9274(26.5)$ & $443(25.6)$ \\
\hline Missing & 4165 (10.6) & $461(21.0)$ \\
\hline \multicolumn{3}{|l|}{ Ever illicit drug use } \\
\hline Yes & $12,093(31.0)$ & $694(32.0)$ \\
\hline No & $26,892(69.0)$ & $1473(68.0)$ \\
\hline Missing & $160(0.4)$ & $21(0.9)$ \\
\hline \multicolumn{3}{|c|}{ Weekly physically active } \\
\hline Yes & $35,283(91.2)$ & $1885(88.0)$ \\
\hline No & $3416(8.8)$ & $256(12.0)$ \\
\hline Missing & $446(1.1)$ & $47(2.1)$ \\
\hline \multicolumn{3}{|c|}{ Number of HPV vaccine doses received } \\
\hline 1 & $1260(3.2)$ & N/A \\
\hline 2 & $4933(12.6)$ & \\
\hline 3 & $32,952(84.2)$ & \\
\hline
\end{tabular}

N/A not applicable

mental health complaints after a median of 5.3 years since vaccination.

The effect of the HPV vaccine on the decrease of incidence of neoplastic changes in the cervical epithelium associated primarily with HPV types 16 and 18 has been regarded as a milestone in cancer prevention. ${ }^{18} \mathrm{~A}$ large body of evidence supported the notion that the HPV vaccine is effective and safe. ${ }^{19,20}$ Follow-up studies of HPV vaccine immunogenicity over the previous decade have suggested that the vaccine effectiveness in the prevention of female genital cancers accounts for $>90 \%$ over at least 10 years, ${ }^{21}$ and projections of cost-effectiveness of vaccination of adolescent girls indicated favorable economic impact. ${ }^{22}$ However, reports of adverse effects, particularly among Danish girls and women, ${ }^{3,23}$ but also among Japanese girls and women, ${ }^{24}$ perpetuated distrust in the vaccine ${ }^{25}$ and subsequent non-compliance to vaccination. $^{2,26}$ Some authors have challenged the quality and completeness of data available to policy makers and stakeholders, ${ }^{27}$ suggesting that changes in HPV immunization policy have not entirely been evidence-based.

We did observe that the fully adjusted model showed a negative association of HPV vaccination with having had sore throat and having been sad. Given the high number of statistical tests performed and in order to lessen the likelihood of chance findings, it may be warranted to adjust the probability level of 0.05 by the number of the tests performed. In this way, the probability level that would be considered statistically significant would be stricter (i.e., the threshold for statistical significance would be considerably lower than 0.05 ). However, safety concerns about medicines and medical devices tested in clinical trials suggest that the acceptance of probability level of 0.05 is recommended because potential adverse effects caused by the tested drug/substance would be more easily detected. ${ }^{28}$ For this reason, and in the light of the HPV vaccine controversy in Denmark, we were compelled to consider the 
Table 2 Associations Between Receiving at Least One Dose of the HPV Vaccine and Physical and Mental Health Complaints 6 Months Prior to Survey Among Girls in Secondary Education Institutions in Denmark $(N=41,333)$

\begin{tabular}{|c|c|c|c|c|c|}
\hline Complaints & $\begin{array}{l}\text { Vaccination } \\
\text { status }\end{array}$ & $\begin{array}{l}\text { Number of girls who } \\
\text { reported the complaint }(\%)\end{array}$ & $\begin{array}{l}\text { Crude model } \\
\text { OR }(95 \% \mathrm{CI})\end{array}$ & $\begin{array}{l}\text { Partially adjusted } \\
\text { model* OR }(95 \% \text { CI) }\end{array}$ & $\begin{array}{l}\text { Fully adjusted model }{ }^{\dagger} \\
\text { OR }(95 \% \text { CI })\end{array}$ \\
\hline \multirow[t]{2}{*}{ Headache } & Vaccinated & $27,769(72.4)$ & $0.96(0.86-1.05)$ & $0.95(0.86-1.06)$ & $0.93(0.82-1.04)$ \\
\hline & Unvaccinated & $1547(73.6)$ & 1.0 (ref) & 1.0 (ref) & 1.0 (ref) \\
\hline \multirow[t]{2}{*}{ Stomachache } & Vaccinated & 18,913 (49.6) & $0.97(0.89-$ & $1.02(0.93-$ & $1.02(0.92-1$ \\
\hline & Unvaccinated & $1056(50.4)$ & 1.0 (ref) & 1.0 (ref) & 1.0 (ref) \\
\hline \multirow[t]{2}{*}{ Menstrual cramps } & Vaccinated & $28,908(75.5)$ & $0.96(0.87-1.07)$ & $1.02(0.92-1.14)$ & $0.97(0.86-1.09)$ \\
\hline & Unvaccinated & $1603(76.1)$ & 1.0 (ref) & 1.0 (ref) & 1.0 (ref) \\
\hline \multirow[t]{2}{*}{ Neck pain } & Vaccinated & $23,482(61.5)$ & $1.00(0.92-1.10)$ & $1.02(0.93-1.12)$ & $1.04(0.94-1.15)$ \\
\hline & Unvaccinated & $1286(61.3)$ & 1.0 (ref) & 1.0 (ref) & 1.0 (ref) \\
\hline \multirow{2}{*}{ Sore throat } & Vaccinated & $19,302(50.6)$ & $0.89(0.81-0.97)$ & $0.89(0.81-0.97)$ & $0.86(0.78-0.95)$ \\
\hline & Unvaccinated & 1125 (53.5) & 1.0 (ref) & 1.0 (ref) & 1.0 (ref) \\
\hline \multirow[t]{2}{*}{ Been sad } & Vaccinated & $28,113(73.4)$ & $0.92(0.83-1.02)$ & $0.89(0.80-0.99)$ & $0.86(0.76-0.97)$ \\
\hline & Unvaccinated & $1580(75.9)$ & 1.0 (ref) & 1.0 (ref) & 1.0 (ref) \\
\hline \multirow{4}{*}{$\begin{array}{l}\text { Been irritable/bad } \\
\text { mood } \\
\text { Been nervous }\end{array}$} & Vaccinated & $33,812(88.2)$ & $1.19(1.05-1.36)$ & $1.09(0.96-1.25)$ & $1.04(0.88-1.22)$ \\
\hline & Unvaccinated & $1816(86.2)$ & 1.0 (ref) & 1.0 (ref) & 1.0 (ref) \\
\hline & Vaccinated & $26,487(69.2)$ & $0.99(0.91-1.10)$ & $0.98(0.89-1.08)$ & $0.95(0.85-1.06)$ \\
\hline & Unvaccinated & $1460(69.3)$ & 1.0 (ref) & 1.0 (ref) & 1.0 (ref) \\
\hline \multirow{2}{*}{$\begin{array}{l}\text { Had sleep } \\
\text { problems }\end{array}$} & Vaccinated & $21,552(56.3)$ & $0.93(0.85-1.02)$ & $0.97(0.88-1.06)$ & $0.96(0.86-1.06)$ \\
\hline & Unvaccinated & $1218(58.0)$ & 1.0 (ref) & 1.0 (ref) & 1.0 (ref) \\
\hline
\end{tabular}

Legend: *Adjusted for age, ethnicity, type of school and living arrangements; ${ }^{\dagger}$ adjusted for variables in partially adjusted model and health behaviors (smoking, alcohol use, drug use and physical activity)

probability level of 0.05 as statistically significant and not perform any corrections of the $p$ value in the final analysis.
Our results are similar to previous large-scale studies involving register-based data that corroborated null association

Table 3 Associations Between the Number of HPV Vaccination Doses Received and Physical and Mental Health Complaints 6 Months Prior to Survey Among Girls in Secondary Education Institutions in Denmark $(N=41,333)$

\begin{tabular}{|c|c|c|c|c|}
\hline Complaints & $\begin{array}{l}\text { Number of HPV vaccine } \\
\text { doses received }\end{array}$ & $\begin{array}{l}\text { Crude model OR } \\
(95 \% \mathrm{CI})\end{array}$ & $\begin{array}{l}\text { Partially adjusted model* OR } \\
\text { (95\% CI) }\end{array}$ & $\begin{array}{l}\text { Fully adjusted model }{ }^{\dagger} \mathrm{OR} \\
(95 \% \mathrm{CI})\end{array}$ \\
\hline \multirow[t]{4}{*}{ Headache } & 0 & $1.0(\mathrm{ref})$ & 1.0 (ref) & 1.0 (ref) \\
\hline & 1 & $1.02(0.87-1.20)$ & $1.01(0.86-1.19)$ & $0.93(0.78-1.11)$ \\
\hline & 2 & $1.02(0.91-1.14)$ & $1.01(0.89-1.13)$ & $0.97(0.85-1.11)$ \\
\hline & 3 & $0.94(0.85-1.04)$ & $0.94(0.85-1.05)$ & $0.92(0.82-1.03)$ \\
\hline \multirow[t]{4}{*}{ Stomachache } & 0 & 1.0 (ref) & 1.0 (ref) & 1.0 (ref) \\
\hline & 1 & $1.06(0.92-1.22)$ & $1.09(0.95-1.26)$ & $1.06(0.90-1.24)$ \\
\hline & 2 & $1.01(0.91-1.12)$ & $1.05(0.94-1.16)$ & $1.03(0.92-1.16)$ \\
\hline & 3 & $0.96(0.88-1.05)$ & $1.01(0.92-1.11)$ & $1.02(0.92-1.13)$ \\
\hline \multirow[t]{4}{*}{ Menstrual cramps } & 0 & 1.0 (ref) & 1.0 (ref) & 1.0 (ref) \\
\hline & 1 & $1.05(0.89-1.24)$ & $1.09(0.92-1.29)$ & $1.00(0.83-1.20)$ \\
\hline & 2 & $0.95(0.84-1.07)$ & $1.00(0.89-1.13)$ & $0.94(0.82-1.08)$ \\
\hline & 3 & $0.96(0.87-1.07)$ & $1.03(0.92-1.14)$ & $0.97(0.86-1.10)$ \\
\hline \multirow{4}{*}{ Neck pain } & 0 & 1.0 (ref) & 1.0 (ref) & 1.0 (ref) \\
\hline & 1 & $1.02(0.88-1.18)$ & $1.03(0.89-1.19)$ & $1.03(0.87-1.21)$ \\
\hline & 2 & $1.05(0.95-1.17)$ & $1.06(0.95-1.18)$ & $1.07(0.95-1.21)$ \\
\hline & 3 & $0.99(0.91-1.09)$ & $1.02(0.93-1.12)$ & $1.04(0.93-1.15)$ \\
\hline \multirow[t]{4}{*}{ Sore throat } & 0 & 1.0 (ref) & 1.0 (ref) & 1.0 (ref) \\
\hline & 1 & $0.96(0.84-1.11)$ & $0.96(0.83-1.11)$ & $0.89(0.76-1.05)$ \\
\hline & 2 & $0.94(0.85-1.04)$ & $0.96(0.84-1.04)$ & $0.90(0.80-1.01)$ \\
\hline & 3 & $0.88(0.80-0.96)$ & $0.88(0.80-0.96)$ & $0.86(0.77-0.95)$ \\
\hline \multirow{4}{*}{ Been sad } & 0 & 1.0 (ref) & 1.0 (ref) & 1.0 (ref) \\
\hline & 1 & $0.96(0.82-1.13)$ & $0.93(0.79-1.10)$ & $0.86(0.72-1.04)$ \\
\hline & 2 & $0.97(0.86-1.09)$ & $0.92(0.81-1.04)$ & $0.89(0.77-1.02)$ \\
\hline & 3 & $0.92(0.83-1.01)$ & $0.88(0.79-0.98)$ & $0.85(0.76-0.97)$ \\
\hline Been irritable/bad & 0 & 1.0 (ref) & 1.0 (ref) & 1.0 (ref) \\
\hline \multirow[t]{3}{*}{$\operatorname{mood}$} & 1 & $1.31(1.05-1.62)$ & $1.24(0.99-1.55)$ & $1.17(0.90-1.52)$ \\
\hline & 2 & $1.15(0.99-1.33)$ & $1.05(0.89-1.22)$ & $1.01(0.84-1.21)$ \\
\hline & 3 & $1.20(1.05-1.36)$ & $1.10(0.96-1.25)$ & $1.04(0.88-1.22)$ \\
\hline \multirow[t]{4}{*}{ Been nervous } & 0 & 1.0 (ref) & 1.0 (ref) & 1.0 (ref) \\
\hline & 1 & $0.99(0.86-1.16)$ & $0.93(0.78-1.10)$ & $0.93(0.78-1.10)$ \\
\hline & 2 & $1.01(0.90-1.13)$ & $0.95(0.84-1.08)$ & $0.95(0.84-1.08)$ \\
\hline & 3 & $0.99(0.90-1.09)$ & $0.94(0.84-1.05)$ & $0.95(0.85-1.06)$ \\
\hline \multirow{4}{*}{ Had sleep problems } & 0 & 1.0 (ref) & 1.0 (ref) & 1.0 (ref) \\
\hline & 1 & $1.04(0.90-1.19)$ & $1.06(0.92-1.23)$ & $1.02(0.87-1.20)$ \\
\hline & 2 & $0.99(0.89-1.10)$ & $1.02(0.92-1.14)$ & $0.99(0.88-1.12)$ \\
\hline & 3 & $0.92(0.84-1.01)$ & $0.96(0.87-1.05)$ & $0.95(0.85-1.05)$ \\
\hline
\end{tabular}

*Adjusted for age, ethnicity, type of school and living arrangements; ${ }^{\dagger}$ adjusted for variables in partially adjusted model and health behavior (smoking, alcohol use, drug use and physical activity) 


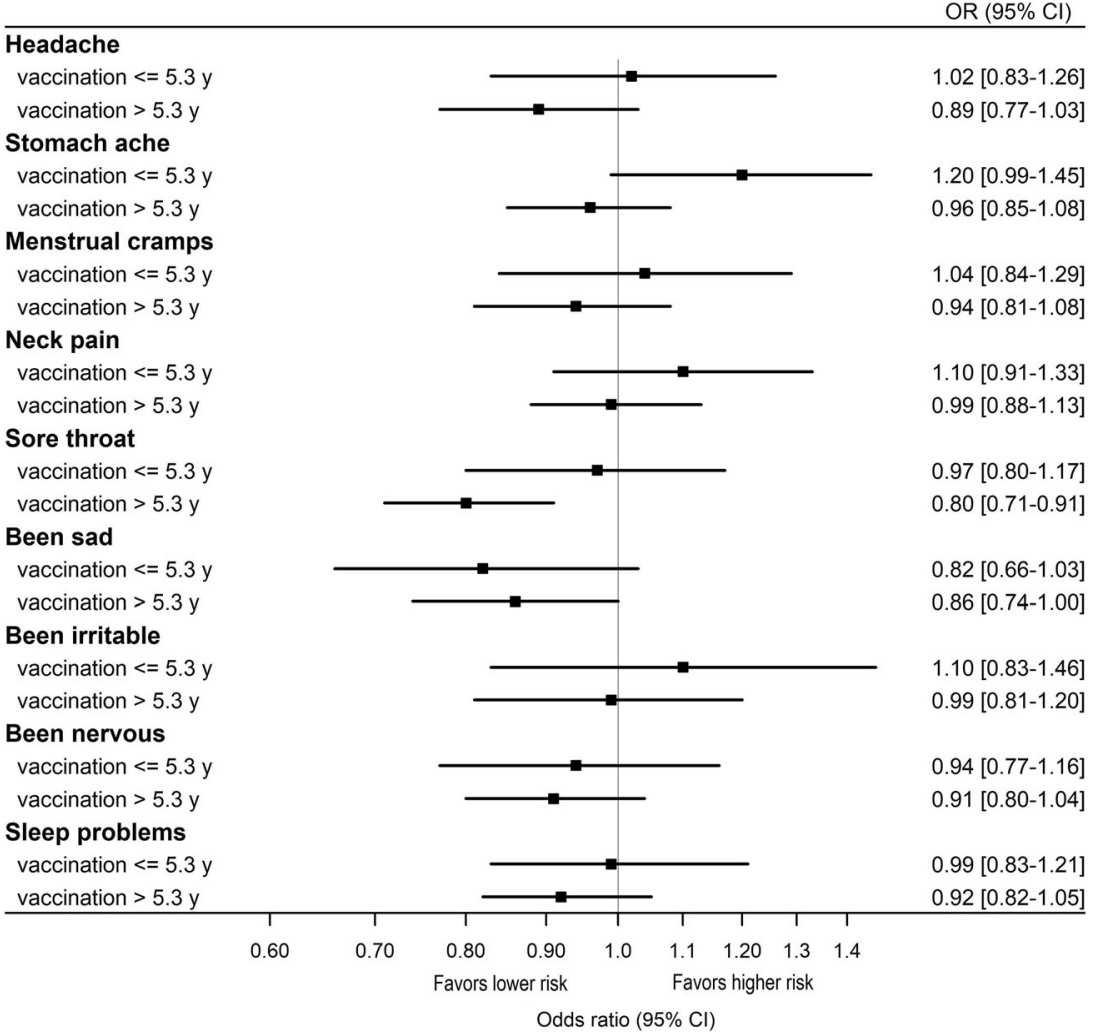

Figure 1 Effect estimates of receiving at least one dose of the HPV vaccine and self-reported physical and mental health complaints in the past 6 months stratified according to median time since vaccination (5.3 years) among girls in secondary education institutions in Denmark (OR, odds ratio; $\mathbf{C I}$, confidence interval).

between HPV vaccination and the onset of different adverse effects in girls. ${ }^{7,27,29,30}$ A study among 240,605 girls aged 11 to 15 years in Finland supported no association between HPV vaccination and unspecified illnesses, complex regional pain syndrome as well as chronic fatigue syndrome. ${ }^{7}$ Register data from the Canadian province of Alberta reported an adverse effect incidence rate of 37.4/100,000 of the administered vaccine doses among close to 200,000 vaccinated girls, which was in accordance with the previously known adverse effect rates. ${ }^{29}$ Similar findings were also observed in the electronic health information system in the Canadian province of Ontario. ${ }^{30}$ Findings from a surveillance system of adverse vaccinal events in Southern Italy found no rare or unexpected vaccine-related health problems. ${ }^{31}$ Moreover, a study in the Netherlands examined the occurrence of a short (3-6 months) and long-term (more than 6 months) fatigue after HPV vaccination and reported that no association was found. ${ }^{32}$

In Denmark, a case-control study using the National Patient Register suggested that females who reported unexpected adverse events following HPV vaccination, such as POTS, fatigue, nausea or neuropathic pain, were more likely to have been treated for a wide array of conditions including infectious and neuro-psychiatric diseases, gastrointestinal or musculoskeletal health issues in the 5-year period before vaccination compared with females who did not report any post-vaccinal events. ${ }^{11}$ Another study also found that the females who reported adverse events had more general practitioner consultations before vaccination. ${ }^{33}$ A case-control study using the DVR found no difference in the presence of psychiatric or somatic disturbances between the females who reported adverse post-vaccinal events and those who did not in a 5-year period since HPV vaccination. ${ }^{34}$ The differences in the results may arise from differences in the sample sizes (1496 vs. 251 females reporting adverse effects) as well as coverage of the registers used in the analysis. ${ }^{35}$ Another analysis of the Danish National Patient Register ${ }^{10}$ supported the notion of higher utilization of health-care service among females who reported adverse events after HPV vaccination.

While the underlying mechanism of the unexpected and non-specific adverse effects after HPV vaccination is not understood, some authors propose a multifactorial approach to the prediction of future susceptibility to adverse effects. ${ }^{36,37}$ It is possible that a complex interplay between individual immune response due to HPV immunization and previous health conditions as well as genetic susceptibility of an individual could play a role in generating the onset of adverse effects of HPV vaccination, particularly that of the autonomic nervous system. ${ }^{36}$ When considering the origins of adverse effects following HPV vaccination, besides an individual's genetic profile, gut microbiome and serum biomarkers could provide a more detailed evidence as to the propensity of having post-vaccination adverse effects. ${ }^{37}$

A major strength of this study was the opportunity to examine different physical and mental health complaints, that 
are not part of health registers data, and cannot, therefore, be studied using a register-based approach. A further strength of this study is the possibility to adjust for a number of covariates that are potentially associated with both HPV vaccination and physical and mental health complaints. The strengths of this study also include a large nationally representative sample of students in secondary education institutions in Denmark. Also, HPV vaccination status was determined based on reliable information from the DVR using the Danish Civil Registration System for precise linkage.

Study limitations include potential information bias, because all analyzed data, except vaccination status, were selfreported. Valid register information on health status is difficult to obtain, because most of the outcomes in this study were non-specific and could have been part of a wide array of diseases and conditions within the patient register. Response rates among all the invited high schools was $87 \%$. Response rates among classes in the participating high schools were $96 \%$ and response rates among students in high schools was $82 \%,{ }^{14}$ which was deemed representative. Participants in the DNYS were more often of Danish origin, of younger age, had parents of higher level of education and income compared with students who did not participate in the survey. ${ }^{14}$ Response rate among students in vocational schools was $69 \%$ and vocational schools from all five Danish regions were represented in the sample. ${ }^{14}$ Compared with high schools, a similar sociodemographic pattern in vocational schools was observed between respondents and non-respondents. Nevertheless, a sample of students from vocational schools included a lower proportion of girls compared with a proportion of girls registered in the vocational schools across Denmark (24\% vs. $40 \%) .{ }^{14}$ Girls who were included in the survey were in a reasonably good health and were not absent from school due to health issues when the survey took place. Therefore, students who were severely ill were not included in the study sample.

We conclude that this study showed that HPV vaccination was not associated with self-reported physical and mental health complaints of students in secondary education institutions after a median of 5.3 years since HPV vaccination. Our study adds to the body of evidence that HPV vaccination is safe and does not cause long-term adverse effects.

Corresponding Author: Janne Schurmann Tolstrup, PhD; National Institute of Public Health University of Southern Denmark, Copenhagen K, Denmark (e-mail: jest@sdu.dk).

Funding Information This study was supported by the Danish Cancer Society (grant no. R163-A10654).

\section{Compliance with Ethical Standards:}

Conflict of Interest: The authors declare that they do not have a conflict of interest.

\section{REFERENCES}

1. Sander BB, Rebolj M, Valentiner-Branth P, Lynge E. Introduction of human papillomavirus vaccination in Nordic countries. Vaccine. 2012; 30:1425-33.

2. Suppli CH, Hansen ND, Rasmussen M, Valentiner-Branth P, Krause TG, Mølbak K. Decline in HPV-vaccination uptake in Denmark - the association between HPV-related media coverage and HPV-vaccination. BMC Public Health. 2018; 18:1360.

3. Brinth LS, Pors K, Theibel AC, Mehlsen J. Orthostatic intolerance and postural tachycardia syndrome as suspected adverse effects of vaccination against human papilloma virus. Vaccine. 2015; 33:2602-5.

4. Hansen PR, Schmidtblaicher M, Brewer NT. Resilience of HPV vaccine uptake in Denmark: Decline and recovery. Vaccine. In press; https://doi. org/10.1016/j.vaccine.2019.12.019.

5. Hviid A, Svanström H, Scheller NM, Grönlund O, Pasternak B, Arnheim-Dahlström L. Human papillomavirus vaccination of adult women and risk of autoimmune and neurological diseases. J Intern Med. 2018; 283:154-65.

6. Feiring B, Laake I, Bakken IJ, et al. HPV vaccination and risk of chronic fatigue syndrome/myalgic encephalomyelitis: A nationwide registerbased study from Norway. Vaccine. 2017; 35:4203-12.

7. Skufca J, Ollgren J, Artama M, Ruokokoski E, Nohynek H, Palmu AA. The association of adverse events with bivalent human papilloma virus vaccination: A nationwide register-based cohort study in Finland. Vaccine. 2018; 36:5926-33.

8. Scheller NM, Pasternak B, Mølgaard-Nielsen D, Svanström H, Hviid A. Quadrivalent HPV Vaccination and the Risk of Adverse Pregnancy Outcomes. N Engl J Med. 2017; 376:1223-33.

9. World Health Organization (WHO). Global Vaccine Safety. Safety update on HPV vaccines. Available at https://www.who.int/vaccine_safety/ committee/topics/hpv/June_2017/en/ Accessed on Apr 1, 2019

10. Mølbak K, Hansen ND, Valentiner-Branth P. Pre-vaccination careseeking in females reporting severe adverse reactions to hpv vaccine. a registry based case-control study. PLoS One. 2016; 11:e0162520.

11. Krogsgaard LW, Bech BH, Plana-Ripoll O, Thomsen RW, Rytter D. Hospital contacts and diagnoses five years prior to HPV vaccination among females referred for suspected adverse vaccine effects: A Danish nationwide case-control study. Vaccine. 2019; 37:1763-8.

12. Michaud PA, Fombonne E. Common mental health problems. BMJ. 2005;330(7495):835-8.

13. Viner R, Christie D. Fatigue and somatic symptoms. BMJ. 2005;330(7498):1012-5.

14. Pisinger V, Mikkelsen SS, Bendtsen P, Egan KK, Tolstrup JS. The Danish National Youth Study 2014: Study design, population characteristics and non-response analysis. Scand $\mathrm{J}$ Public Health, in press https://doi.org/10.1177/1403494817729283

15. Grove Krause T, Jakobsen S, Haarh M, Mølbak K. The Danish vaccination register. Euro Surveill. 2012; 17:1-6.

16. European Union Agency for Fundamental Rights. Consent to use data on children. Available from https://fra.europa.eu/en/publication/2017/ mapping-minimum-age-requirements/use-consent. Accessed 17 Jan 2020.

17. Gordon M, Lumley T. Forestplot: Advanced Forest Plot Using 'grid' Graphics. Available from https://cran.r-project.org/web/packages/forestplot/index.html. Accessed 24 Sept 2019.

18. Lowy DR. HPV vaccination to prevent cervical cancer and other HPVassociated disease: from basic science to effective interventions $\mathrm{J}$ Clin Invest. 2016; 126:5-11.

19. Maver PJ, Poljak M. Progress in prophylactic human papillomavirus (HPV) vaccination in 2016: A literature review. Vaccine. 2018; 36:541623.

20. Garcia-Perdomo HA, Osorio JC, Fernandez A, Zapata-Copete JA, Castillo A. The effectiveness of vaccination to prevent the papillomavirus infection: a systematic review and meta-analysis. Epidemiol Infect. 2019; 147:e156.

21. Kjaer SK, Nygård M, Dillner J, et al. A 12-Year Follow-up on the LongTerm Effectiveness of the Quadrivalent Human Papillomavirus Vaccine in 4 Nordic Countries. Clin Infect Dis. 2018; 66:339-45.

22. Brisson $\mathbf{M}$, Laprise JF, Drolet $\mathbf{M}$, et al. Comparative costeffectiveness of the quadrivalent and bivalent human papillomavirus vaccines: a transmission-dynamic modeling study. Vaccine. 2013; 31:3863-71.

23. Cramon C, Poulsen CL, Hartling UB, Holden IK, Johansen IS. Possible adverse effects of the quadrivalent human papillomavirus vaccine in the 
Region of Southern Denmark: a retrospective, descriptive cohort study. Dan Med J. 2017; 64:1-6.

24. Ozawa K, Hineno A, Kinoshita T, Ishihara S, Ikeda SI. Suspected Adverse Effects After Human Papillomavirus Vaccination: A Temporal Relationship Between Vaccine Administration and the Appearance of Symptoms in Japan. Drug Saf. 2017; 40:1219-29.

25. Beppu H, Minaguchi $M$, Uchide $\mathbf{K}$, Kumamoto $\mathbf{K}$, Sekiguchi $\mathbf{M}$, Yaju Y. Lessons learnt in Japan from adverse reactions to the HPV vaccine: a medical ethics perspective. Indian J Med Ethics. 2017; 2:82-8.

26. Larson HJ, Wilson R, Hanley S, Parys A, Paterson P. Tracking the global spread of vaccine sentiments: the global response to Japan's suspension of its HPV vaccine recommendation. Hum Vaccin Immunother. 2014; 10:2543-50.

27. Jørgensen L, Doshi P, Gøtzsche P, Jefferson T. Challenges of independent assessment of potential harms of HPV vaccines. BMJ. 2018; 362:k3694.

28. European Agency for the Evaluation of Medicinal Products (2002). Committee for Proprietary Medicinal Products. Points to consider on multiplicity issues in clinical trials. Available from https://www.ema. europa.eu/en/documents/scientific-guideline/points-consider-multiplicity-issues-clinical-trials_en.pdf. Accessed 17 Jan 2020.

29. Liu XC, Bell CA, Simmonds KA, Svenson LW, Russell ML. Adverse events following HPV vaccination, Alberta 2006-2014. Vaccine. 2016; 34:1800-5.

30. Harris T, Williams DM, Fediurek J, Scott T, Deeks SL. Adverse events following immunization in Ontario's female school-based HPV program. Vaccine. 2014; 32:1061-6.
31. Scavone C, Di Mauro C, Brusco S, et al. Surveillance of adverse events following immunization related to human papillomavirus vaccines: 12 years of vaccinovigilance in Southern Italy. Expert Opin Drug Saf. 2019; 18:427-33.

32. Schurink-Van't Klooster TM, Kemmeren JM, van der Maas NAT, et al. No evidence found for an increased risk of long-term fatigue following human papillomavirus vaccination of adolescent girls. Vaccine. 2018; 36:6796-802.

33. Krogsgaard LW, Vestergaard $\mathbf{C H}$, Plana-Ripoll O, et al. Health care utilization in general practice after HPV vaccination-A Danish nationwide register-based cohort study. PLoS One. 2017; 12:e0184658.

34. Ulendorf Jacobsen S, Valentiner-Branth P, Mølbak K. Examining determinants for reporting suspected adverse events following HPV vaccination in Denmark. Vaccine. 2018; 36:6158-62.

35. Lynge E, Sandegaard JL, Rebolj M. The Danish National Patient Register. Scand J Public Health. 2011; 39(7 Suppl):30-3.

36. Blitshteyn S, Brinth L, Hendrickson JE, Martinez-Lavin M. Autonomic dysfunction and HPV immunization: an overview. Immunol Res. 2018; 66:744-54.

37. Campbell-Tofte J, Vrahatis A, Josefsen $\mathbf{K}$, Mehlsen $\mathbf{J}$, Winther $\mathbf{K}$. Investigating the aetiology of adverse events following HPV vaccination with systems vaccinology. Cell Mol Life Sci. 2019; 76:67-87.

Publisher's Note: Springer Nature remains neutral with regard to jurisdictional claims in published maps and institutional affiliations. 